\section{AVERTIN NARCOSIS."}

\section{BY}

GEORGE EDWARDS, M.R.C.S., I.R.C.P.,

ANAESThetist, PRINCE OF WALES's GeNeral hospital, totTenHaM, THE SAMARITAN FREE HOSPITAL, AND THE ITALIAN HOSPITAL, LONDON.

Avertin has been used in this country for the last year and $a$ half and has been on the market for the last six months. During the last vear I have been privileged to work with Dr. J. Blomfield and Dr. Z. Mennell, and was able to gain some knowledge of its use.

Avertin is tribromethylalcohol, a white crystalline solid. It is soluble in water to the extent of $3 \frac{1}{2}$ per cent. at body temperature. When heated above $40^{\circ}$ Centigrade or exposed to light for long it decomposes into dibromacetaldehrde and hrdrobromic acid. The former substance is extremely irritating to the bowel ; the hydrobromic acid is readily observed by its reaction with Congo red reagent and prorides a ready and necessary means for testing the purity of avertin solutions.

Introduced into the rectum avertin is readily absorbed from its solution. It is excreted almost entirely by the liver and kidneys, and appears in the urine in combination with glycuronic acid. Straub ${ }^{1}$ has recovered from the urine as much as 81 per cent. of the dose given.

The anaesthetic properties of avertin are best indicated by the fact that the chemical manufacturers now call it "the basal narcotic." Full surgical anaethesia can be obtaired, and is occasionally obtained by avertin alone, but the variation in depth of anaesthesia produced by similar doses on differcnt patients is so great that one cannot set out definitely to produce anaesthesia solely by this method. Once a patient is narcotized with avertin, only a very small quautity of inhalation anaesthetic is required to complete the anaesthesia. From the anaesthetist's point of view this is the chief merit of the drug. The patient has much more cause to welcome it. The anaesthetic is given in the ward without fuss, the falling asleep is gradual and peaceful, and the awakening equally. so. Wven though the narcosis has been very light, there will be no memory of anything from the time of the injection. Further, the erents of the next twenty-four or thirty-six hours will not be remembered with any clearness, and the whole horrible business of being anaesthetized and of recovering is thus not only diminished in fact, but is minimized in retrospect. The merits of avertin narcosis are, in short, those of rectal anaesthesia combined with those of deep preliminary and subsequent narcosis.

Avertin is found to be of particular value in dealing with highly nervous patients, with cases of Graves's disease, and, I am told, with children. In patients with respiratory affections it largely reduces the amount of inhalation ancesthetic required, or may enable the operations necessary to be carried out under local anaesthesia. Flessa ${ }^{2}$ at the Munich Gynaccological Clinic has used light avertin narcosis in demonstrating patients to his classes. He finds that the patients are not sensible of their exposure, and that the true pain reflexes persist whilst the functional element is obliterated, so that a much more accurate diagnosis is possible. For obstetric purposes, avertin was at first said to be unsuitable, owing to its paralysing action upon plain muscle. However, reports vary exceedingly.

The conditions in which avertin is contraindicated are such as one would expect from its method of absorption and excretion. All inflammatory and ulcerated conditions of the rectum and colon rule it out. Cases with severe renal and hepatic lesions should not be given avertin, although nephrectomy has been performed and cases with severe jaundice have been operated upon under it. Acidosis, advanced cachexia, and serious blood diseases are also given as contraindications. Recent work in Germany by Pribram $^{3}$ shows that patients with hyperthyroidism can take massive doses of avertin and can eliminate them quickly. This suggests that patients with deficient thyroid secretion will not eliminate the drug, and that one line of treatment in case of delayed return to consciousness would

* A paper read in the Section of Anaesthetics at the Annual Meeting of the British Medical Association, Manchester, 1929. be the injection of thyroxin. Further, in cases where there is doubt as to the advisability of giving avertin, a determination of the basal metabolism may help in coming to a reasoned decision.

The physiological changes in avertin narcosis are a falling of the blood pressure and a decrease in the respiratory movements. No definite changes in the pulse rate have been observed. The fall in the blood pressure is usually within the limits of that observed in deep sleep, between 10 and $30 \mathrm{~mm}$. I have never seen more than this change, and tiere appear to be no reports of a greater fall which could have been duc to the avertin alone. The respiratory centre is affected and a slight cranosis is usually observed, more particularly in patients who have had preliminary doses of morphine. The breathing is shailow and the rate normal or slightly quickened. Patients vary a great deal in the amount of cyanosis they show; much depends, of course, upon the general condition of the patient, upon the anatomy of the air passages, and apon the dose of avertin they have had. Marked cyancsis will not occur if the dose has not been greater than 0.1 gram per kilo of body weight, and as long as the airway is kept clear.

The anaesthesia comes on gradually and takes from twenty to thirty minutes to reach completion. The ocular reflexes disappear in the usual way, and with the routine dosage the patients end up with contracted pupils which react to light, with an absent conjunctival reflex, and with no, or wnly a slight, corneal reflex. There may still, however, be a skin reflex, and pinching the inner side of the thigh or scratching with a needle may cause a movement. A sidden blast of strong ether vapour may produce a cough; this is a useful preliminary test to give one an idea of how much inhalation anaesthetic will be needed. With increasing experience of a routine dosage it is possible fairly, accurately to judge what state of anaesthesia has been effected, and to estimate how much pushing of the inhalation anaesthetic will be necessary.

The actual administration of avertin is now a simple proceeding. In the first cases, the weighing out of the requisite amount of powder, the slow dissolving of it in water at $38^{\circ} \mathrm{C}$., the testing of the solution, the giving of two rectal washouts before the administration and one after the operation, together with the peculiar anxiety regarding the patient, made an avertin anaesthesia a formidable business. Finding that more often than not a complete anaesthesia did not result, the anaesthetist felt that a great deal of trouble had been taken for no reasonable return. The introduction of a standard concentrated arertin solution ready for dilution has simplified matters, and experience has shown that much lavage of the rectum is not only unnecessary but undesirable, as an excess of fluid in the bowel may interfere with absorption.

The preliminary measures are an enema the night before operation, repeated next morning if it should seem necessary. In emergencies, satisfactory results have been obtained without any preparation of the howel. ${ }^{4}$ Some observers think it good to give a sedative overnight. The desirability of an injection of morphine or of omnopon before the rectal injection appears to be a moot point. Acting upon Dr. Mennell's suggestion, I tried a series of cases with and without omnopon. The difference in the degree of anaesthesia was definite, but the general condition of the patients without morphine was better: respirations were less shallow and there was less cyanosis. As it had been agreed by this time that full anaesthesia was not to be attempted with avertin alone, the omission of the morphine merely slightly added to the amount of ether or chloroform required.

The dosage of avertin is still a matter for discussion; but the general agreement has been that where there is no marked weakness or general infection, 0.1 gram per kilo of body weight is a perfectly safe dose, and this is the dose which is largely used; 0.125 and 0.15 gram per kilo have been used with success, but where there has been trouble it has nearly always been with these high doses. In Germany larger doses still have been tried. ${ }^{5}$ I have not been able to get the full results, but am told that cases of Graves's disease stand tḩse doses particularly well. 
Avertin is provided in a concentrated solution with amylene hydrate-1 gram in $1 \mathrm{c.cm}$. The dose works out at between 6 and 8 grams. The appropriate amount of the solution is measured out and enough distilled water to make a $2 \frac{1}{2}$ or 3 per cent. solution is lieated to $38^{\circ} \mathrm{C}$. The avertin is then added and the mixture is shaken till a clear solution results. The most important points are that the temperature must never exceed $40^{\circ} \mathrm{C}$. and that the final solution must be free from hydrobromic acid and therefore free from dibromacetaldehyde; $5 \mathrm{c.cm}$. of the dilute solution are taken and a drop of 1 in 1,000 Congo red solution is added. The cotour should remain bright orange red. The blue reaction of free acid is sometimes delayed and it is essential to make the test immediately before administration.

The solution being now ready and satisfactory, it is injected into the rectum fairly slowly through a catheter and funnel. The catheter must be passed well into the rectum proper. The patient is best arranged lying on the left side with a pillow under the pelvis. The 250 or $350 \mathrm{c.cm}$. of solution are easily retained if the injection takes three or four minutes. Attempts have been made to give fractional doses, running in more solution if the anaesthesia produced by the original dose was inadequate. It has been shown that the arertin in solution is more readily absorbed than the water. This makes all calculations with regard to strength of solution useless, and it is impossible to gauge rate of absorption or to prophesy the depth of anaesthesia. which will be obtained. If undue lepth should be observed at an early stage, wasing out the rectum for the purpose of removing any of the avertin that may remain there is a sound proceeding.

In practice, it seems best to give the rectal injection thirty minutes before the advertised time of the operation. This means that the patient need not be disturbed for twenty minutes, and then may be taken straight to the theatie. During the induction period we have endeavoured to keep the patient quiet, to keep the room dark, and to keep out all sources of irritation. Flessa, however, says that some patients resent strongly the sepulchral atmosphere, and that light and noise have no effect upon the induction, so that it is better to allow the ordinary ncise and disturbances to go on normally. 6

Having taken the patient to the theatre, the anaesthetist tests the skin reflexes, and either starts the inhalation anaesthetic at once or, if a fairly deep state has been achieved, withbolds the ether till necessars. Ether, C.E. mixture, chloroform, gas and oxygen, or local anaesthetic may be used. If gas and oxygen be used, one has to wait for a severe stimulus to be given by the surgeon before the patient will breathe sufficiently deeply to take in enough of the gases. The amount of ether and chloroform used will be measured in drachms rather than in ounces. Once a sufficient relaxation has been obtained, it is often unnecessary to give any more of the inhalant. The peritoneum that has been relaxed enough for the abdomen to be opened will remain so for closing, unless the operation lasts so long that the avertin effect is wearing off. The usual duration of the narcosis is from one and a half to two hours from the time of the injection. About four hours from the start the patient is usually quite conscious of his surroundings.

The chief care in the nursing is watching the relaxed jaw and tongue; this applies during the period of induction as well as of recovery. A rectal washout used to be given on return to the ward. This is now considered to be unnecessary. Should the patient's condition demand it the routine saline treatment is carried out.

In only one case have I scen any trouble directly due to an avertin anaesthesia. In this case, which was reported in the Lancet ${ }^{7}$ by Sir Francis Shipway and Dr. Blomfield, the patient died twenty hours after the administration of a dose at the rate of 0.125 gram per kilo. It would seem probable that in that case a deficiency of thyroid secretion had diminished the paticnt's power of detoxicating and eliminating the drug.

The lines of treatment in cases of prolonged delay in return to consciousness are respiratory stimulation by injections of lobeline, the giving of carbon dioxide and oxygen mixture, and injections of ephedrine to maintain the circulation. So far as I can discover, thyroxin has not been tried; but this would seem a rational method of assisting elimination.

In conclusion, avertin can be used to provide a deep narcosis, which is casily convertible into an anaesthesia by the giving of very small quantities of the ordinary anaesthetics. It is reasonably safe in doses up to $0.1 \mathrm{gram}$ per kilo of body weight. It eliminates nearly all the mental stress which accompanies the usual administration of an anaesthetic. It has no effect upon the respiratory tract. It is well taken by persons with excessive thyroid secretion. It is fairly easy to give.

On the other hand, it is not controllable and cannot safely be used as the sole anaesthetic agent. It has to be prepared with care. It should not be used where there is any disease of the kidneys, liver, or colon, or where there is thyroid deficiency. It is not, as was at first hoped, an anaesthetic that anybody can give at any time. It requires an experienced anaesthetist to control the superimposed anaesthesia. The necessity of giving the injection half an hour beforchand adds to the anaesthetist's duties and makes its use diffcult in hospital practice where cascs follow each other with rapidity.

In certain cases and types of patients avertin is a great help. As a routine anaesthetic agent, it is hardly practicable.

1 Klin. Woch. 1928, No. 49. ${ }^{2}$ M Müch. med. Woch., June 7th, 1929.

3 Zentraibl. f. Chir., 1929, No. 19. 4 Miinch. med. Woch., June 7th, 1929.
5 Zentralbl. f. Chir., 1929, No. 19. 6 Münch. med. Woch., June 7th, 1929.
; 1929, vol. i, p. 546.

5 Zentralbl. f. Chir., 1929, No. 19. 6 Münch. med. Woch., June 7th, 1929.

\section{THE TREATIENT OF TORTICOLLIS.*}

\section{BY}

B. WHITCHURCH HOWELL, F.R.C.S.ENG.,

SURGEON TO THE QUEEN'S HOSPITAL FOR CHILDREN, LONDON; SURGEON TO THE BROOKFIELD ORTHOPAEDIC HOSPITAL; ORTHOPAEDIC SURGEON TO THE ESSEX COUNTY COUNCIL.

TonticolnIs or wry-neck is a very rare deformity, occurring once in every 150,000 births. Congenital club-foot is ten times as frequent. The outstanding deformity is the tilting of the head towards the affected side, with rotation of the chin forward and away from the affected side. Secondary deformities are facial asymmetry and scoliosis, chiefly of the cervical region.

The cause of the deformity is contraction of the sternnmastoid muscle, more particularly its sternal head. Its origin is either congenital or acquired, the following being the chief varieties:

A. Congenital.-(1) Prenatal; (2) natal.

B. Acquired.-(1) Rheumatic; (2) reflex-for example, tubercle of cervical spine; (3) ocular; (4) spasmodic, etc.

The congenital variety, as indicated abore, has two subdivisions-prenatal and natal-and there are several theories as to their origin.

1. Prenatal.-The prenatal subdivision inclucles those due to abnormal posture in utero, giving rise, on the one hand, to temporary or permanent shortening of the sterno-mastoid, or, on the other, to localized fibrosis and contracture of the muscle from interference with the blood supply-thus being allied to Volkmann's ischaemic paralysis. The rarer forms are due to abnormal development of the cervical vertebrae, which may be unusual in number and often wedged. A certain percentage of the latter are associated with Sprengel's shoulder or congenital eleration of the shoulder. In addition, cervical ribs may be present. The contracture of the sterno-mastoid is usually on the right side, and is very rarely bilateral. I have personal records of three cases of bilateral torticollis, each one of which was associated with congenital abnormalities of -the cervical spine, whilst in one Sprengel's deformity was present.

2. Natal.-The natal subdivision of the congenital varicty is generally associated with a difficult confinement. The presentation is usually a breech, when there may

* A paper read in the Section of Orthopaedies at the Annual Mecting of the British Medical Association at Manchester on July 25th. This was illustrated by photographs of patieñts before, during, and after treat ment. Photographs of the operation table used are reproduced at page 721 . 